\title{
16 Erhebungsmaterial
}

\subsection{Fragebogen für die Schüler und Schülerinnen}

Fragebogen für die Schüler und Schülerinnen

Nationalfondsprojekt 'Textproduktionsspezifische Schriftsprachentwicklung'. Leitung: Prof. Dr. Regula Schmidlin, Mitarbeiterin: Pascale Schaller, Universität Freiburg

๑ Open Access. ( 2018 Pascale Schaller, publiziert von De Gruyter. (๕) BY-NC-ND Dieses Werk ist lizenziert unter der Creative Commons Attribution-NonCommercial-NoDerivatives 4.0 Lizenz. https://doi.org/10.1515/9783110555165-016 


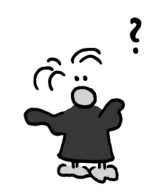

Lieber Schüler, liebe Schülerin

Auf den folgenden Seiten findest du verschiedene Fragen, die uns interessieren. Bei einigen Fragen musst du ein Kreuz oder mehrere Kreuze machen. Dort hat es Kästchen. Bei anderen Fragen sollst du etwas hinschreiben. Dort ist eine schreibende Hand abgedruckt.

Bitte lies die Anweisungen gut durch. Lies alle möglichen Antworten, bevor du dich für eine oder mehrere entscheidest. Lass bitte keine Frage aus.

Es gibt keine richtigen oder falschen Antworten, schreib einfach, was für dich stimmt. Du kannst jederzeit fragen, wenn du etwas nicht verstehst.

Wir brauchen deine Antworten für ein Forschungsprojekt. Sie werden anonymisiert, d.h. dein Name wird durch eine Nummer ersetzt. So bleiben die Angaben "geheim" und werden an niemanden weitergegeben. Beantworte die Fragen darum möglichst ehrlich.

Vielen Dank für deine Hilfe! 
Fragen zu dir

1. Vorname

2. Name

3. Bist du ein Mädchen oder ein Junge?

$\square_{1}$ ein Mädchen

$\square_{2}$ ein Junge

4. Dein Geburtsdatum

5. In welchem Land bist du geboren?
$\square_{1}$ in der Schweiz
$\square_{2}$ in einem anderen Land:
in welchem?

6. Wenn du nicht in der Schweiz geboren bist, seit wann lebst du in der Schweiz?

(z.B.: Seit ich zwei Jahre alt bin.)

7. Welche Sprache sprecht ihr normalerweise zuhause? Wenn es verschiedene sind, kreuze sie alle an.

$\square_{1}$ Schweizerdeutsch
$\square_{2}$ Hochdeutsch
$\square_{3}$ Französisch
$\square_{4}$ Italienisch
$\square_{5}$ Spanisch
$\square_{6}$ eine oder mehrere andere Sprachen:
welche?

你 
8. Welche Sprache hast du in deiner Familie zuerst gelernt (Muttersprache)? Wenn du zwei- oder mehrsprachig aufgewachsen bist, gib beide/alle Sprachen an.

9. Wenn Schweizerdeutsch nicht deine Muttersprache ist: Wann hast du begonnen, es zu lernen? $\square_{1}$ Schweizerdeutsch

$\square_{2}$ Hochdeutsch

$\square_{3}$ Französisch

$\square_{4}$ Italienisch

$\square_{5}$ Spanisch

$\square_{6}$ eine oder mehrere andere Sprachen: welche?

$\square_{1}$ bevor ich 6 Jahre alt war

$\square_{2}$ etwa mit 6 bis 9 Jahren

$\square_{3}$ später

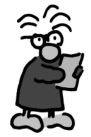

\section{Fragen zum Fach Deutsch}

In der Tabelle stehen verschiedene Sätze. Kreuze jeweils die Antwort an, die für dich stimmt. Es gibt kein richtig oder falsch. Mach pro Zeile ein Kreuz.

10.

\begin{tabular}{|l|c|c|c|c|}
\hline & stimmt gar nicht & $\begin{array}{c}\text { stimmt eher } \\
\text { nicht }\end{array}$ & stimmt eher & stimmt genau \\
\hline $\begin{array}{l}\text { 1. Das Fach Deutsch ha- } \\
\text { be ich gern. }\end{array}$ & $\square_{1}$ & $\square_{2}$ & $\square_{3}$ & $\square_{3}$ \\
\hline $\begin{array}{l}\text { 2. Im Fach Deutsch lerne } \\
\text { ich schnell. }\end{array}$ & $\square_{1}$ & $\square_{2}$ & $\square_{3}$ & $\square_{4}$ \\
\hline $\begin{array}{l}\text { 3. Im Fach Deutsch er- } \\
\text { reiche ich gute Leistun- } \\
\text { gen. }\end{array}$ & $\square_{1}$ & $\square_{2}$ & $\square_{3}$ & $\square_{4}$ \\
\hline $\begin{array}{l}\text { 4. Im Fach Deutsch be- } \\
\text { komme ich gute Noten. }\end{array}$ & $\square_{1}$ & $\square_{2}$ & $\square_{3}$ & $\square_{4}$ \\
\hline $\begin{array}{l}\text { 5. Das Fach Deutsch ist } \\
\text { eines meiner Lieblings- } \\
\text { fächer. }\end{array}$ & $\square_{1}$ & $\square_{2}$ & \\
\hline
\end{tabular}




\section{Fragen zum Schreiben}

In der Tabelle stehen verschiedene Sätze. Kreuze jeweils die Antwort an, die für dich stimmt. Es gibt kein richtig oder falsch. Mach pro Zeile ein Kreuz.

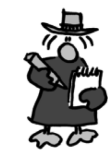

11.

\begin{tabular}{|l|c|c|c|c|}
\hline & stimmt gar nicht & $\begin{array}{c}\text { stimmt eher } \\
\text { nicht }\end{array}$ & stimmt eher & stimmt genau \\
\hline $\begin{array}{l}\text { 1. In der Schule schreibe } \\
\text { ich besonders gern. }\end{array}$ & $\square_{1}$ & $\square_{2}$ & $\square_{3}$ & $\square_{3}$ \\
\hline $\begin{array}{l}\text { 2. Schreiben kann ich } \\
\text { gut. }\end{array}$ & $\square_{1}$ & $\square_{2}$ & $\square_{3}$ & \\
\hline $\begin{array}{l}\text { 3. Das Schreiben von } \\
\text { Texten fällt mir beson- } \\
\text { ders leicht. }\end{array}$ & $\square_{1}$ & $\square_{2}$ & $\square_{3}$ & \\
\hline $\begin{array}{l}\text { 4. Wenn ich wünschen } \\
\text { könnte, würde ich ger- } \\
\text { ne öfter Texte und Auf- } \\
\text { sätze schreiben. }\end{array}$ & $\square_{1}$ & $\square_{2}$ & $\square_{4}$ \\
\hline $\begin{array}{l}\text { 5. Für meine Texte be- } \\
\text { komme ich gute Noten. }\end{array}$ & $\square_{1}$ & $\square_{2}$ & $\square_{3}$ & \\
\hline
\end{tabular}

12. Was sind deine Stärken im Schreiben? Beschreibe bitte möglichst genau. 
13. Womit hast du beim Schreiben eher Probleme? Beschreibe bitte möglichst genau.

14. Was für Texte schreibst du am liebsten? Warum? 


\section{Du und der Computer}

In den Tabellen stehen verschiedene Sätze, die den Computer betreffen (Laptops zählen auch dazu). Kreuze jeweils die Antwort an, die für dich stimmt. Es gibt kein richtig oder falsch. Mach pro Zeile ein Kreuz.

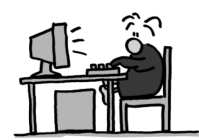

15.

15.
\begin{tabular}{|l|c|c|c|c|}
\hline & stimmt gar nicht & $\begin{array}{c}\text { stimmt eher } \\
\text { nicht }\end{array}$ & stimmt eher & stimmt genau \\
\hline $\begin{array}{l}\text { 1. Ich arbeite gerne am } \\
\text { Computer. }\end{array}$ & $\square_{1}$ & $\square_{2}$ & $\square_{3}$ & $\square_{3}$ \\
\hline $\begin{array}{l}\text { 2. Ich kann den Compu- } \\
\text { ter gut bedienen. }\end{array}$ & $\square_{1}$ & $\square_{2}$ & $\square_{3}$ & $\square_{3}$ \\
\hline $\begin{array}{l}\text { 3. Computer finde ich } \\
\text { eine gute Erfindung. }\end{array}$ & $\square_{1}$ & $\square_{2}$ & $\square_{4}$ \\
\hline $\begin{array}{l}\text { 4. Computer erleichtern } \\
\text { uns das Leben. }\end{array}$ & $\square_{1}$ & $\square_{2}$ & $\square_{3}$ & $\square_{4}$ \\
\hline $\begin{array}{l}\text { 5. Mit dem Computer } \\
\text { umzugehen, finde ich } \\
\text { schwierig. }\end{array}$ & $\square_{1}$ & $\square_{2}$ & $\square_{3}$ & \\
\hline $\begin{array}{l}\text { 6. Für mich ist das Be- } \\
\text { dienen eines Computers } \\
\text { leicht. }\end{array}$ & $\square_{1}$ & $\square_{2}$ & $\square_{4}$ \\
\hline
\end{tabular}

16.

\begin{tabular}{|l|c|c|c|c|}
\hline & stimmt gar nicht & $\begin{array}{c}\text { stimmt eher } \\
\text { nicht }\end{array}$ & stimmt eher & stimmt genau \\
\hline $\begin{array}{l}\text { 1. Ich schreibe Aufsätze } \\
\text { lieber auf dem Compu- } \\
\text { ter als von Hand. }\end{array}$ & $\square_{1}$ & $\square_{2}$ & $\square_{3}$ & \\
\hline $\begin{array}{l}\text { 2. Ich finde das Schrei- } \\
\text { ben auf einer Tastatur } \\
\text { schwierig. }\end{array}$ & $\square_{1}$ & $\square_{2}$ & $\square_{3}$ & \\
\hline $\begin{array}{l}\text { 3. Ich schreibe ohne } \\
\text { grosse Probleme auf } \\
\text { der Tastatur. }\end{array}$ & $\square_{1}$ & $\square_{2}$ & $\square_{3}$ \\
\hline $\begin{array}{l}\text { 4. Texte schreiben dau- } \\
\text { ert bei mir auf dem } \\
\text { Computer länger als } \\
\text { von Hand. }\end{array}$ & $\square_{1}$ & $\square_{2}$ & $\square_{4}$ \\
\hline $\begin{array}{l}\text { 5. Ich finde, das Texte- } \\
\text { schreiben auf dem } \\
\text { Computer ist anstren- } \\
\text { gender als von Hand. }\end{array}$ & $\square_{1}$ & $\square_{2}$ & $\square_{3}$ & \\
\hline
\end{tabular}




\title{
Dein Zuhause
}

In den letzten Fragen geht es um dein Zuhause. Wenn du an verschiedenen Orten lebst, also z.B. manchmal bei deinem Vater und manchmal bei deiner Mutter, dann nimm den Ort, an dem du am häufigsten wohnst. Mach bitte pro Frage nur ein Kreuz.

17. Wie viele Bücher habt ihr zuhause? Zeitschriften und Heftli sollst du nicht mitzählen.

$$
\begin{aligned}
& \square_{1} \text { keine oder fast keine } \\
& \square_{2} \text { ein Tablar voll } \\
& \square_{3} \text { zwei Tablare voll } \\
& \square_{4} \text { ein Büchergestell voll } \\
& \square_{5} \text { zwei Büchergestelle voll } \\
& \square_{6} \text { mehr als zwei Büchergestelle voll }
\end{aligned}
$$

18. Habt ihr Computer zuhause? Zähle auch Laptops dazu.

\author{
$\square_{1}$ ja, einen \\ $\square_{2}$ ja, mehrere \\ $\square_{3}$ nein, keinen
}

19. Hast du einen eigenen Computer zuhause?

$\square_{1}$ ja, einen nur für mich

$\square_{2}$ nein, aber einen, den ich mit Geschwistern oder Eltern teile.

20. Wie viel Zeit verbringst du zuhause durchschnittlich am Computer?

$\square_{1}$ weniger als eine halbe Stunde pro Tag

$\square_{2}$ ungefähr eine halbe Stunde pro Tag

$\square_{3}$ ungefähr eine Stunde pro Tag

$\square_{4}$ mehr als eine Stunde pro Tag 


\section{Wie würdest du 'Sprache' erklären?}

21.

Stell dir eine Begegnung mit einem ausserirdischen Wesen vor, das für ganz kurze Zeit hier auf der Erde vorbeischaut. Es weiss nichts davon, wie wir Menschen sind, wie wir leben und wie wir miteinander umgehen. Nur für einen ganz kurzen Moment kann es uns überhaupt verstehen. Das Wesen interessiert sich dafür, wie wir miteinander kommunizieren. Erkläre ihm, was 'Sprache' für dich ist und wie du sie dir vorstellst... 


\subsection{Fragebogen für die Eltern}

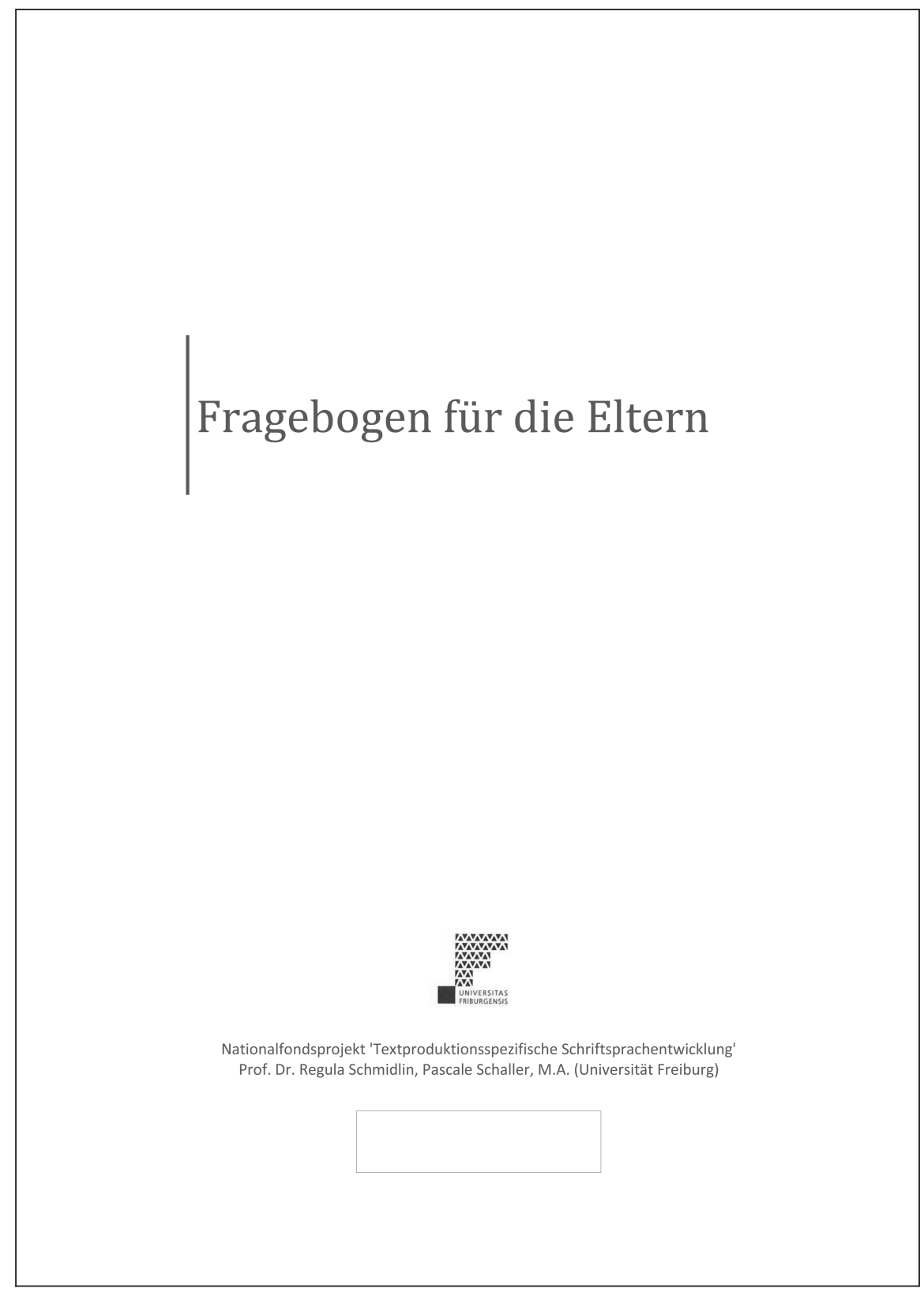


UNIVERSITÉ DE FRIBOURG SUISSE

UNIVERSITÄT FREIBURG SCHWEIZ

Sehr geehrte Eltern

Im beiliegenden Schreiben haben wir Sie über ein Nationalfondsprojekt zur Schriftsprachentwicklung informiert, an dem auch die Klasse Ihres Kindes beteiligt ist. Sie finden im vorliegenden Dokument einige Fragen zu Ihnen und zu Ihrem Kind, für deren Beantwortung wir uns herzlich bei Ihnen bedanken. Sie leisten damit einen wichtigen Beitrag zu unserer Studie.

Wenn Sie auf den folgenden Seiten jeweils als „Mutter“ oder „Vater“ angesprochen werden, dann gilt diese Bezeichnung auch für Bezugspersonen, die mit dem Kind zusammenleben.

Es gibt für die aufgeführten Fragen keine erwünschten oder unerwünschten, richtigen oder falschen Antworten, sondern es geht um Ihre persönliche Meinung und Einschätzung. Für eine ehrliche Beantwortung bedanken wir uns daher bereits im Voraus.

Wir bitten Sie, diese Hinweise zu befolgen:

- Beantworten Sie die Fragen der Reihe nach und lassen Sie keine Frage aus.

- Machen Sie Kreuze nur in und nicht zwischen die Kästchen.

Selbstverständlich werden wir Ihre Angaben ausschliesslich in anonymisierter Form verwenden und Ihren Namen nach Abgabe des Bogens durch ein Kürzel ersetzen. Weder werden Rückschlüsse auf Sie möglich sein, noch werden die Daten an Dritte weitergegeben, auch nicht an die Schule oder die Lehrpersonen.

Wir bitten Sie, den Fragebogen zusammen mit der unterschriebenen Einverständniserklärung Ihrem Kind im beigelegten verschlossenen Umschlag in die Schule mitzugeben. Die Lehrperson wird die Umschläge an uns weiterleiten.

Wir bedanken uns für Ihr Interesse und Ihre Mitarbeit! 
Angaben zu Ihrer Person

1. Vorname und Name

2. In welcher Beziehung stehen Sie zum Kind?

$$
\begin{aligned}
& \square_{1} \text { Mutter } \\
& \square_{2} \text { Vater } \\
& \square_{3} \text { Stiefmutter } \\
& \square_{4} \text { Stiefvater } \\
& \square_{5} \text { Pflegemutter } \\
& \square_{6} \text { Pflegevater } \\
& \square_{7} \text { Grossmutter } \\
& \square_{8} \text { Grossvater } \\
& \square_{9} \text { andere: }
\end{aligned}
$$

3. Vorname und Name des Kindes, das den Fragebogen nach Hause gebracht hat

4. Wie häufig haben Sie und Ihr Partner zusammen Ihrem Kind vorgelesen, als es noch nicht lesen konnte?

$$
\begin{aligned}
& \square_{1} \text { fast täglich } \\
& \square_{2} \text { einmal oder mehrmals pro Woche } \\
& \square_{3} \text { einmal oder mehrmals pro Monat } \\
& \square_{4} \text { weniger als einmal pro Monat } \\
& \square_{5} \text { selten }
\end{aligned}
$$


Fragen zu beiden Eltern / Bezugspersonen des Kindes

Die folgenden Fragen betreffen jeweils beide Eltern oder Bezugspersonen des Kindes.

Wenn Sie alleinerziehende Mutter oder alleinerziehender Vater sind, kreuzen Sie das bitte hier an und beantworten Sie die folgenden Fragen nur bezogen auf Sie persönlich:

$\square_{1}$ Ich bin alleinerziehende Mutter.

$\square_{2}$ Ich bin alleinerziehender Vater.

5. Welche Schule haben Sie zuletzt abgeschlossen? Bitte geben Sie nur Ihren höchsten Schulabschluss an.

\begin{tabular}{l|c|c}
\hline & Mutter & Vater \\
\hline 1. keinen Schulabschluss & $\square_{1}$ & $\square_{11}$ \\
\hline 2. Abschluss der obligatorischen Schule (9 Schuljahre) & $\square_{2}$ & $\square_{12}$ \\
\hline $\begin{array}{l}\text { 3. Berufslehre / Berufsschule / Handelsschule / Diplommittel- } \\
\text { schule }\end{array}$ & $\square_{3}$ & $\square_{13}$ \\
\hline 4. Maturitätsschule / Gymnasium / Lehrerseminar & $\square_{4}$ & $\square_{14}$ \\
\hline 5. Fachhochschule / Pädagogische Hochschule / Technikum & $\square_{5}$ & $\square_{15}$ \\
\hline 6. Universität / ETH & $\square_{6}$ & $\square_{16}$ \\
\hline 7. andere: & $\square_{7}$ & $\square_{17}$ \\
\hline
\end{tabular}

\section{Sind Sie zurzeit erwerbstätig?}

\begin{tabular}{l|c|c}
\hline & Mutter & Vater \\
\hline Ja, ich bin zurzeit erwerbstätig. & & \\
\hline 1. Ich bin Vollzeit angestellt. & $\square_{1}$ & $\square_{11}$ \\
\hline 2. Ich bin Teilzeit angestellt. & $\square_{2}$ & $\square_{12}$ \\
\hline Nein, ich bin zurzeit nicht erwerbstätig. & & \\
\hline 3. Ich bin auf Stellensuche. & $\square_{3}$ & $\square_{13}$ \\
\hline 4. Ich bin beurlaubt (z.B. Mutterschaftsurlaub). & $\square_{4}$ & $\square_{14}$ \\
\hline 5. Ich bin erwerbsunfähig infolge von Krankheit / Unfall. & $\square_{5}$ & $\square_{15}$ \\
\hline 6. Ich befinde mich in Ausbildung, Weiterbildung, Umschulung. & $\square_{6}$ & $\square_{16}$ \\
\hline 7. Ich bin Hausfrau / Hausmann. & $\square_{7}$ & $\square_{17}$ \\
\hline 8. Ich bin pensioniert (Vorruhestand, Ruhestand). & $\square_{8}$ & $\square_{18}$ \\
\hline
\end{tabular}


7. Welchen Beruf üben Sie im Moment aus? Bitte geben Sie den Beruf möglichst genau an.

> Falls Sie derzeit nicht erwerbstätig sind, geben Sie den Beruf an, den Sie zuletzt ausgeübt haben.

Beruf der Mutter:

Beruf des Vaters:

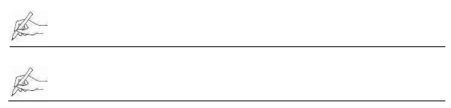

8. Wenn Sie einer Erwerbstätigkeit nachgehen: Welche Funktion haben Sie derzeit inne?

\begin{tabular}{l|c|c}
\hline & Mutter & Vater \\
\hline 1. Direktorin, Direktor & $\square_{1}$ & $\square_{11}$ \\
\hline $\begin{array}{l}\text { 2. Leitende Angestellte / Beamtin, leitender Angestellter / Beam- } \\
\text { ter (höheres Kader) }\end{array}$ & $\square_{2}$ & $\square_{12}$ \\
\hline $\begin{array}{l}\text { 3. Angestellte / Beamtin, Angestellter / Beamte mit Vorge- } \\
\text { setztenfunktion (mittleres Kader) }\end{array}$ & $\square_{3}$ & $\square_{13}$ \\
\hline $\begin{array}{l}\text { 4. Angestellte / Beamtin, Angestellter / Beamte in Expertenfunk- } \\
\text { tion }\end{array}$ & $\square_{4}$ & $\square_{14}$ \\
\hline 5. Arbeiterin / Beamtin, Arbeiter / Beamte & $\square_{5}$ & $\square_{15}$ \\
\hline 6. Selbstständig erwerbend & $\square_{6}$ & $\square_{16}$ \\
\hline 7. andere: & $\square_{7}$ & $\square_{17}$ \\
\hline f
\end{tabular}

9. Sind Sie anderen bei der Arbeit vorgesetzt? Wie viele Personen arbeiten nach Ihren Anweisungen?

\begin{tabular}{l|c|c}
\hline & Mutter & Vater \\
\hline 1. keine & $\square_{1}$ & $\square_{11}$ \\
\hline 2. 1-10 Personen & $\square_{2}$ & $\square_{12}$ \\
\hline 3. mehr als 10 Personen & $\square_{3}$ & $\square_{13}$ \\
\hline
\end{tabular}


Fragen zu Ihrem Kind

10. Wurden die Sprachkompetenzen Ihres Kindes irgendwann von einer Fachperson (Logopädin/Logopäde, Kinderarzt/Kinderärztin usw.) abgeklärt?

$\square_{1}$ Nein, es wurde keine Abklärung gemacht. $\Rightarrow$ Sie sind am Ende des Fragebogens angelangt.

Ja, unser Kind wurde abgeklärt. $\Rightarrow$ Bitte beantworten Sie die Fragen 11 bis 15 .

11. Wenn ja: Von wem wurde Ihr Kind abgeklärt?

12. In welchem Alter wurde Ihr Kind abgeklärt?

13. Aus welchen Gründen wurde Ihr Kind abgeklärt?

然

14. Was war der Befund der Abklärung?

15. Besuchte/besucht Ihr Kind regelmässig eine Fachperson?

$\square$ Nein, es gab nach der Abklärung keine weiteren Sitzungen. $\Rightarrow$ Sie sind am Ende des Fragebogens angelangt.

$\square_{2}$ Ja. $\Rightarrow$ Bitte beantworten Sie die Fragen 16 und 17.

16. Wenn ja: Von was für einer Fachperson wurde/wird Ihr Kind betreut?

17. Während/seit wie vielen Schuljahren besuchte/besucht Ihr Kind die Fachperson?

Herzlichen Dank für Ihre Angaben! 